\title{
Type 1 Acute Aortic Dissection in the Early Period After COVID-19 Infection
}

\author{
Mesut Engin ${ }^{1}$, Ufuk Aydın ${ }^{1}$, Hacı Eskici ${ }^{1}$, Yusuf Ata ${ }^{1}$, Tamer Türk ${ }^{1}$ \\ 1. Cardiovascular Surgery, University of Health Sciences, Bursa Yuksek Ihtisas Training and Research Hospital, Bursa, \\ TUR \\ Corresponding author: Mesut Engin, mesut_kvc_cor@hotmail.com
}

\begin{abstract}
The coronavirus disease 2019 (COVID-19) pandemic has resulted in over 105 million confirmed cases and over 2.3 million deaths globally as of February 3, 2021. Cardiovascular problems due to COVID-19 infection include acute coronary syndrome (due to coronary occlusion, thrombosis), myocardial damage without coronary artery disease, arrhythmias, heart failure, pericardial effusion, and thromboembolic events. A 62year-old male patient was admitted to our emergency department with a complaint of chest pain radiating to his back. The patient had a history of hospitalization for seven days in the outpatient clinic and 10 days in the intensive care unit due to COVID-19 infection with severe lung involvement. In contrast-enhanced thoracoabdominal CT, a dissection line starting from the ascending aorta and progressing to the iliac bifurcation was observed. Ascending aorta and transverse arch replacement was performed with a $30-\mathrm{mm}$ polytetrafluoroethylene tube graft. The patient was discharged home 15 days postoperatively.
\end{abstract}

Categories: Cardiac/Thoracic/Vascular Surgery, Infectious Disease

Keywords: coronavirus/covid-19, cardiovascular system, pandemic, inflammation, aortic dissection

\section{Introduction}

Type 1 acute aortic dissection (AAD) is a serious clinical condition that requires urgent surgical intervention as it is associated with high mortality and morbidity. Causes such as atherosclerotic foci, degeneration, and tissue diseases may play a role in the emergence of this condition [1]. The global pandemic caused by coronavirus disease 2019 (COVID-19) has been around for about a year and the disease still maintains its severity. Increased inflammation due to this virus can lead to various cardiovascular diseases such as cardiac damage, thrombosis, and cardiomyopathy [2].

Inflammation plays an important role in vascular plaque rupture. Therefore, it is known to play a role in the development of AAD [3]. In addition, a case of arteriopathy related to COVID-19 infection has been reported in a pediatric case [4]. In this case report, we present the successful surgical treatment of a patient with type $1 \mathrm{AAD}$ in the early period after the COVID-19 infection.

Review began 03/01/2021 Review ended 03/05/2021 Published 03/07/2021

\section{(c) Copyright 2021}

Engin et al. This is an open access article distributed under the terms of the Creative Commons Attribution License CC-BY 4.0., which permits unrestricted use, distribution, and reproduction in any medium, provided the original author and source are credited.

\section{Case Presentation}

A 62-year-old male patient was admitted to our emergency department with a complaint of chest pain radiating to his back. A dissection line starting from the ascending aorta was observed in the contrastenhanced thoracoabdominal CT, and hence the patient was transferred to the intensive care unit (ICU). On physical examination, peripheral pulses of the lower and upper extremities were palpable. The arterial blood pressure measured in both arms was normal and similar (right arm: 170/90 mmHg; left arm: 175/90 mmHg). His past medical history was notable for chronic atrial fibrillation, diabetes mellitus, and lower extremity deep venous insufficiency. There was no history or sign of any connective tissue disease. The current medical treatment of the patient included insulin, calcium dobesilate, low molecular weight heparin, and beta-blocker. The patient had a history of hospitalization for seven days in the outpatient clinic and 10 days in the ICU due to COVID-19 infection with severe lung involvement (Figures $1 \mathrm{~A}, 1 \mathrm{~B}, 1 \mathrm{C}$ ), with his polymerase chain reaction (PCR) being positive. The patient had been discharged at 20 days. Additionally, he had received steroid treatment (methylprednisolone $16 \mathrm{mg} /$ day) due to ongoing respiratory symptoms. 


\section{Cureus}

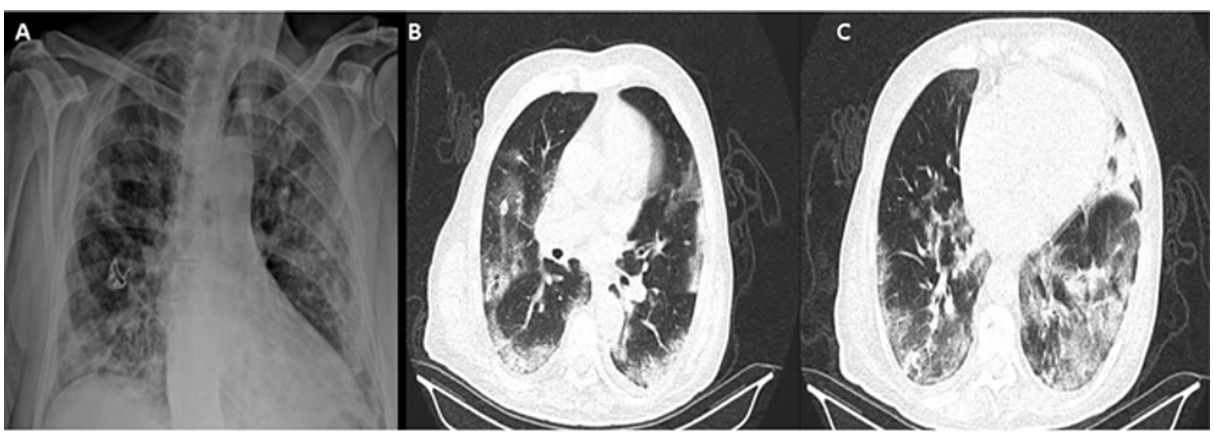

FIGURE 1: (A) Previous chest radiogram, (B, C) previous axial thorax tomography images of the patient with COVID-19 infection

COVID-19: coronavirus disease 2019

He was evaluated with chest radiography (Figure $2 A$ ), transthoracic echocardiography, and thoracoabdominal CT tests. In contrast-enhanced thoracoabdominal CT, a dissection line starting from the ascending aorta and progressing to the iliac bifurcation was observed (Figures $2 B, 2 C, 2 D, 2 E$ ). Also, sequela images of lung involvement areas due to COVID-19 infection were observed on CT images (Figure $2 F$ ). On transthoracic echocardiography, the ejection fraction was $60 \%$, and a dissection flap in the ascending aorta was observed. The left ventricular end-diastolic diameter was $62 \mathrm{~mm}$, the end-systolic diameter was $43 \mathrm{~mm}$, and the left atrial diameter was $52 \mathrm{~mm}$. No pathological finding was observed in the heart valves.

Preoperative blood parameters were as follows: lactate dehydrogenase: $417 \mathrm{U} / \mathrm{L}$; aspartate aminotransferase: 15; alanine aminotransferase: 10; albumin: $39.2 \mathrm{~g} / \mathrm{L}$; urea: $22 \mathrm{mg} / \mathrm{dL}$; creatinine: $1.19 \mathrm{mg} / \mathrm{dL}$; white blood cells: $11.2610^{3} / \mu \mathrm{L}$; neutrophils: $10.0410^{3} / \mu \mathrm{L}$; lymphocytes: $0.6110^{3} / \mu \mathrm{L}$; hematocrit: $40 \%$; and platelets: $17810^{3} / \mu \mathrm{L}$.

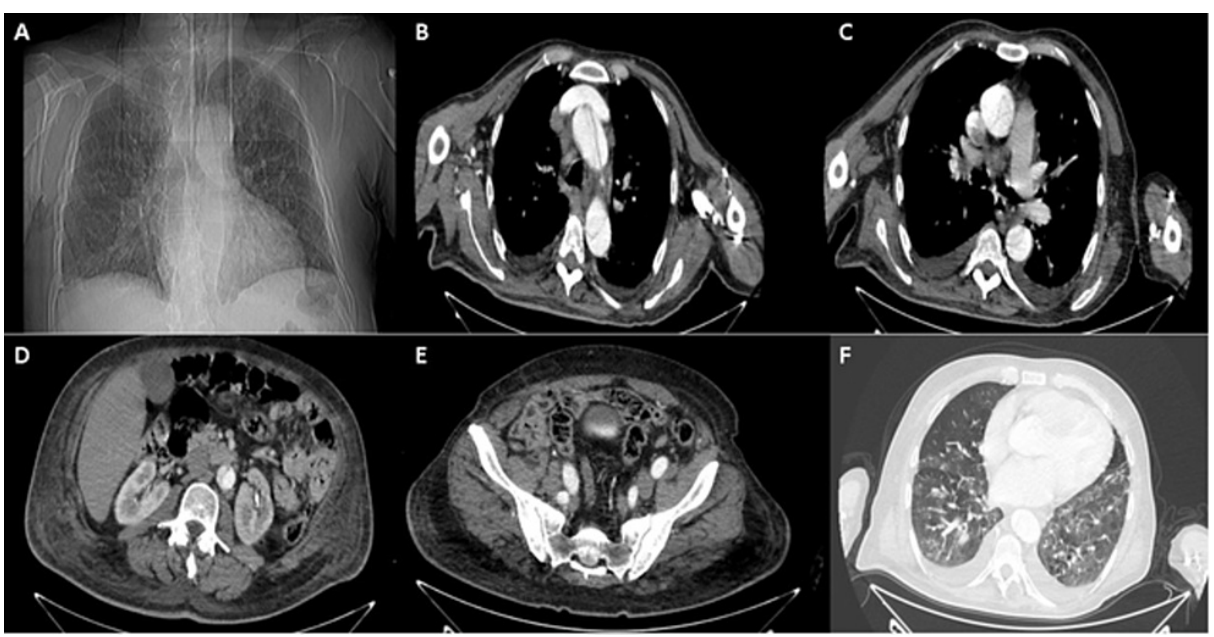

FIGURE 2: (A) Current chest radiogram, (B, C) current axial contrastenhanced thoracoabdominal CT images of the patient, (D, E) a dissection line progressing to the iliac bifurcation, $(F)$ axial thorax tomography images of the patient with sequela images of lung involvement areas due to COVID-19 infection

CT: computed tomography; COVID-19: coronavirus disease 2019

A cardiopulmonary bypass with right axillary artery-venous two-stage cannulation and moderate hypothermia $\left(28^{\circ} \mathrm{C}\right)$ was performed on the patient emergently and under general anesthesia after he underwent a median sternotomy. Heparinization (300 IU/kg) produced an active clotting time of over 400 seconds. The ascending aorta was isolated (Figure $3 \mathrm{~A}$ ). A left atrial vent was placed. Cardiac arrest was achieved with cold blood cardioplegia (BC) $(10-15 \mathrm{~mL} / \mathrm{kg}$, approximately $1,000 \mathrm{ml})$, given both antegrade and retrograde. Cardiac arrest was sustained with BC (300 ml), which was applied every 15-20 minutes. An aortotomy was performed and both the ascending aorta and transverse arch were replaced with a 30-mm 
polytetrafluoroethylene tube graft (Figure 3B). The distal anastomosis was performed under selective antegrade cerebral perfusion (20 minutes) with moderate hypothermia. The heart was successfully defibrillated. Cardiopulmonary bypass was weaned with low-dose inotrope support. Surgical incisions were closed by placing tube drains. The patient was admitted to the cardiovascular surgical ICU after the operation; a chest radiogram was obtained (Figure 3 C), and the patient received standard postoperative care. Following the provision of hemodynamic stability, extubation was performed at the 36th hour postoperatively. He was discharged home 15 days postoperatively.

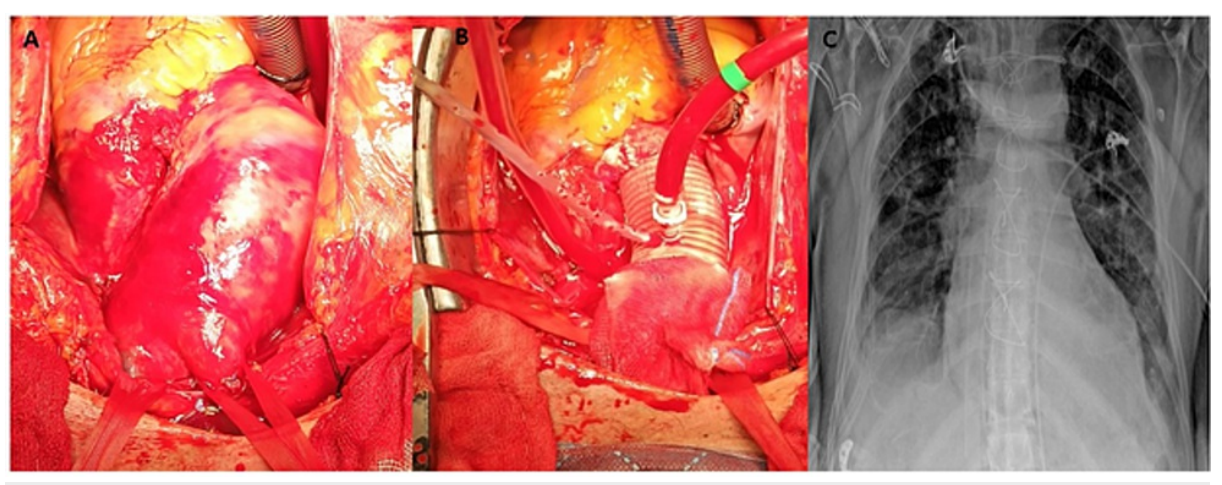

FIGURE 3: (A) Operative image of the dissected ascending aorta, (B) image of the aortic graft, $(\mathrm{C})$ early postoperative chest radiogram image

\section{Discussion}

In this current case report, we shared the experience related to the surgical treatment of type $1 \mathrm{AAD}$ in a patient who had contracted COVID-19 within a month beforehand but had only mild respiratory symptoms. The effects of this infectious disease, which has impacted the whole world for about a year, on the cardiovascular system are emerging day by day.

Inflammation plays an important role in the development of aortic dissection, as found in many patients. Although dissection may occur due to collagen tissue diseases, it is often caused by the rupture of an atherosclerotic plaque as the blood passes through the intima-media layers and progresses distally. Also, inflammation plays an important role in this plaque rupture [1]. Serum proinflammatory cytokines are elevated in coronavirus cases, resulting in lung damage and an increase in microthrombotic events [5]. Furthermore, neutrophil infiltration has been demonstrated in lung tissues in COVID-19 cases during autopsies [6].

Cardiovascular problems resulting from coronavirus infection encompass acute coronary syndrome (due to coronary occlusion, thrombosis), myocardial damage without coronary artery disease, arrhythmias, heart failure, pericardial effusion, and thromboembolic events [7]. In our literature review, we did not find a case report that covers the early period after COVID-19 infection in a patient during the ongoing pandemic. However, serious renal and cardiac damages are associated with severe COVID-19 infections, and they significantly increase the mortality rates [8].

He et al. have reported about four type $1 \mathrm{AAD}$ cases admitted to their clinic in the initial phase of the pandemic. Although the authors did not have a definitive diagnosis in these cases, they observed suspicious inflammatory changes in the lung images. The operative team implemented the necessary preventive measures and successfully performed the operations [9]. However, in these cases, there is no information about the COVID-19 PCR positivity, and the patients were evaluated as potential COVID-19 cases only due to preoperative suspicion raised by the lung images. A similar case with these findings was reported by Akgul et al. [10]. Our case is differentiated from these cases with the finding of a positive PCR one month prior to the patient's current presentation and the observation of an aortic dissection during the recovery period.

\section{Conclusions}

The ongoing COVID-19 epidemic represents a unique healthcare crisis in the history of mankind. Hence, it is currently not possible to fully predict the problems that may be caused by COVID-19. In our case, we think that the inflammatory process caused by COVID-19 may have been a triggering factor for the development of an aortic dissection. Further studies are required to obtain confirmation regarding this hypothesis.

\section{Additional Information}

\section{Disclosures}


Human subjects: Consent was obtained or waived by all participants in this study. N/A issued approval N/A. The written informed consent was obtained from the patient. . Conflicts of interest: In compliance with the ICMJE uniform disclosure form, all authors declare the following: Payment/services info: All authors have declared that no financial support was received from any organization for the submitted work. Financial relationships: All authors have declared that they have no financial relationships at present or within the previous three years with any organizations that might have an interest in the submitted work. Other relationships: All authors have declared that there are no other relationships or activities that could appear to have influenced the submitted work.

\section{References}

1. Oz K, Iyigun T, Karaman Z, Çelik Ö, Akbay E, Akınc O, Erkanli K: Prognostic value of neutrophil to lymphocyte ratio and risk factors for mortality in patients with Stanford type A aortic dissection. Heart Surg Forum. 2017, 20:E119-23. 10.1532/hsf.1736

2. Kir D, Beer N, De Marchena EJ: Takotsubo cardiomyopathy caused by emotional stressors in the coronavirus disease 2019 (COVID-19) pandemic era. J Card Surg. 2021, 36:764-9. 10.1111/jocs.15251

3. Han L, Dai L, Zhao YF, et al.: CD40L promotes development of acute aortic dissection via induction of inflammation and impairment of endothelial cell function. Aging (Albany NY). 2018, 10:371-85. 10.18632/aging.101394

4. Gulko E, Overby P, Ali S, Mehta H, Al-Mufti F, Gomes W: Vessel wall enhancement and focal cerebral arteriopathy in a pediatric patient with acute infarct and COVID-19 infection. AJNR Am J Neuroradiol. 2020, 41:2348-50. 10.3174/ajnr.A6778

5. Wang D, Hu B, Hu C, et al.: Clinical characteristics of 138 hospitalized patients with 2019 novel coronavirus-infected pneumonia in Wuhan, China. JAMA. 2020, 323:1061-9. 10.1001/jama.2020.1585

6. Yao XH, Li TY, He ZC, et al.: A pathological report of three COVID-19 cases by minimal invasive autopsies (Article in Chinese). Zhonghua Bing Li Xue Za Zhi. 2020, 49:411-7. 10.3760/cma.j.cn112151-2020031200193

7. Hendren NS, Drazner MH, Bozkurt B, Cooper LT Jr: Description and proposed management of the acute COVID-19 cardiovascular syndrome. Circulation. 2020, 141:1903-14. 10.1161/CIRCULATIONAHA.120.047349

8. Bonow RO, Fonarow GC, O'Gara PT, Yancy CW: Association of coronavirus disease 2019 (COVID-19) with myocardial injury and mortality. JAMA Cardiol. 2020, 5:751-3. 10.1001/jamacardio.2020.1105

9. He H, Zhao S, Han L, et al.: Anesthetic management of patients undergoing aortic dissection repair with suspected severe acute respiratory syndrome COVID-19 infection. J Cardiothorac Vasc Anesth. 2020, 34:1402-5. 10.1053/j.jvca.2020.03.021

10. Akgul A, Turkyilmaz S, Turkyilmaz G, Toz H: Acute aortic dissection surgery in a patient with COVID-19 . Ann Thorac Surg. 2021, 111:e1-3. 10.1016/j.athoracsur.2020.06.005 